\title{
A Rectilinearity Measurement for Polygons
}

\author{
Joviša Žunič^ and Paul L. Rosin \\ Computer Science, Cardiff University, \\ Queen's Buildings, Newport Road, PO Box 916, \\ Cardiff CF24 3XF, Wales, U.K. \\ \{J.Zunic, Paul.Rosin\}@cs.cf.ac.uk
}

\begin{abstract}
In this paper we define a function $\mathcal{R}(P)$ which is defined for any polygon $P$ and which maps a given polygon $P$ into a number from the interval $(0,1]$. The number $\mathcal{R}(P)$ can be used as an estimate of the rectilinearity of $P$. The mapping $\mathcal{R}(P)$ has the following desirable properties:

- any polygon $P$ has the estimated rectilinearity $\mathcal{R}(P)$ which is a number from $(0,1]$;

- $\mathcal{R}(P)=1$ if and only if $P$ is a rectilinear polygon, i.e., all interior angles of $P$ belong to the set $\{\pi / 2,3 \pi / 2\}$;

- $\inf _{P \in \Pi} \mathcal{R}(P)=0$, where $\Pi$ denotes the set of all polygons;

- a polygon's rectilinearity measure is invariant under similarity transformations.

A simple procedure for computing $\mathcal{R}(P)$ for a given polygon $P$ is described as well.
\end{abstract}

Keywords: Shape, polygons, rectilinearity, measurement.

\section{Introduction}

Shape plays an important part in the processing of visual information, and is actively being investigated in a wide spectrum of areas, from art [13] through to science [3]. Within computer vision there have been many applications of shape to aid in the analysis of images, and standard shape descriptors include compactness, eccentricity [12], circularity [4], ellipticity [9], and rectangularity [11].

This paper describes a shape measure that has received little attention: rectilinearity. While there exists a variety of approaches to computing the related measure of rectangularity [11], rectilinearity covers a wider space of shapes since the number of sides of the model shape is variable. It is only required that the angles of a rectilinear polygon belong to the set $\{\pi / 2,3 \pi / 2\}$. This means that it is not convenient to fit the model 1 to the data and measure the discrepancies between the two, which is the approach that is often applied to compute compactness and rectangularity.

\footnotetext{
* J. Žunić is also with the Mathematical Institute of Serbian Academy of Sciences, Belgrade.

${ }^{1}$ Fitting a rectilinear shape is possible, as demonstrated by Brunn et al. [1], but is complex, and potentially unreliable and inaccurate. Our proposed approach avoids fitting, and is therefore simpler and faster.
} 
Our motivation in developing a rectilinearity shape measure is to provide a useful tool for the analysis of buildings in aerial photographs. Over the last 10-20 years there has been considerable research in this area with the goal of providing automatic photo interpretation which would be particularly useful for cartographers $[2|5| 6 \mid 8]$. Such systems are typically based on perceptual grouping of edges and incorporate additional information such as shadows, knowledgebased rules, as well as shape (typically rectangular). Since many buildings appear rectilinear from an overhead view then such a shape measure could be used in a hypothesis and test paradigm to filter out unlikely candidates which have inappropriate shapes.

Let us conclude this introduction by noticing that a variety of rectilinearity measures for polygons which are based only on a measure of the angles of the considered polygon can be derived very easily. But such a defined rectilinearity measure would imply that the polygons with the same angles have the same estimated rectilinearity which is not always acceptable (see Fig. 1).
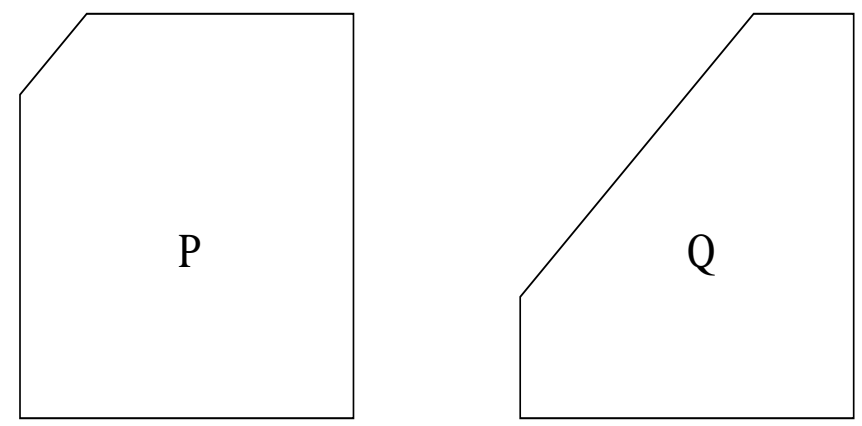

Fig. 1. Two given 5-gons have identical angles, but it is natural to expect that $P$ should have a higher estimated rectilinearity than $Q$.

\section{Definitions and Basic Statements}

A polygon $P$ is rectilinear if its interior angles belong to the set $\{\pi / 2,3 \pi / 2\}$ (see Fig. 2 for an example). In this paper we define a rectilinearity measure for arbitrary polygons. Precisely, we define a mapping $\mathcal{R}(P)$ which maps a given polygon $P$ into a number from the interval $(0,1]$.

We will use the following denotations (see Fig. 3 and Fig. 4 for some illustrations).

The set of all polygons will be denoted by $\Pi$. For a given $n$-gon $P$ having vertices denoted by $A_{0}, A_{1}, \ldots, A_{n-1}, A_{n}=A_{0}$, its edges will be denoted $e_{i}=$ $\left[A_{i-1}, A_{i}\right]$ for $i=1,2, \ldots, n$. The Euclidean length of the straight line segment $e=\left[\left(x_{1}, y_{1}\right),\left(x_{2}, y_{2}\right)\right]$ is $l_{2}(e)=\sqrt{\left(x_{1}-x_{2}\right)^{2}+\left(y_{1}-y_{2}\right)^{2}}$, while the length of $e$ according to the $l_{1}$ metric is $l_{1}(e)=\left|x_{1}-x_{2}\right|+\left|y_{1}-y_{2}\right|$. 


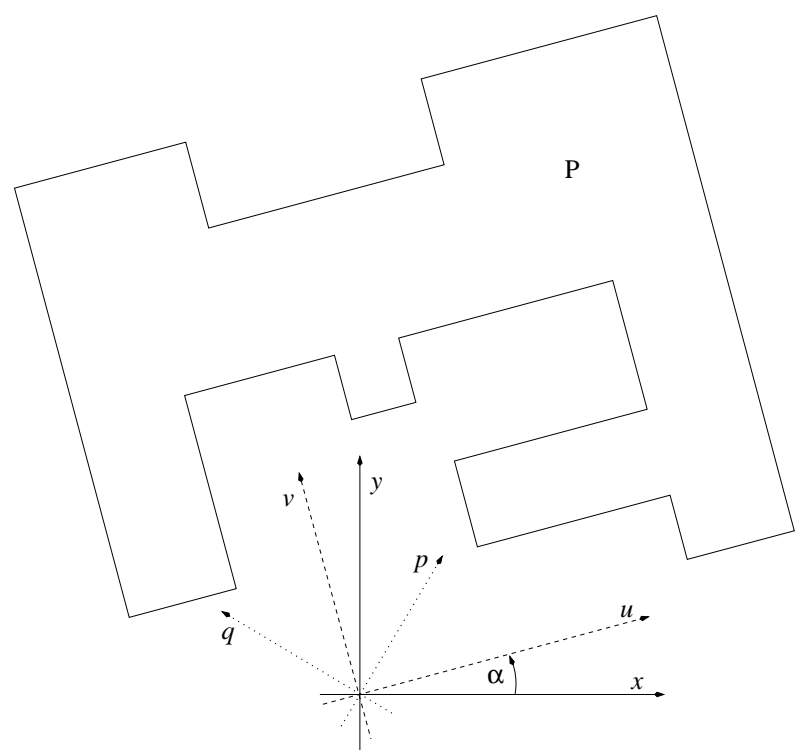

Fig. 2. For the given rectilinear 20-gon $P$, its $l_{1}$ perimeter $\mathcal{P}_{1}(P)$ has the minimum value if the coordinate axes are chosen to be parallel with $u$ and $v$. This minimum value corresponds to $\mathcal{P}_{1}(P,-\alpha)$ if $x$ and $y$ are taken to be the coordinate axes.

$\mathcal{P}_{2}(P)$ will denote the Euclidean perimeter of $P$, while $\mathcal{P}_{1}(P)$ will denote the perimeter of $P$ in the sense of $l_{1}$ metrics. So,

$$
\mathcal{P}_{2}(P)=\sum_{e_{i} \text { is an edge of } P} l_{2}\left(e_{i}\right) \text { and } \quad \mathcal{P}_{1}(P)=\sum_{e_{i} \text { is an edge of } P} l_{1}\left(e_{i}\right) .
$$

Since isometric polygons do not necessarily have the same perimeter under the $l_{1}$ metric, we shall use $\mathcal{P}_{1}(P, \alpha)$ for the $l_{1}$ perimeter of the polygon which is obtained by the rotating $P$ by the angle $\alpha$ with the origin as the centre of rotation. If the same rotation is applied to the edge $e$, the $l_{1}$ perimeter of the obtained edge will be denoted as $l_{1}(e, \alpha)$.

If the oriented angle between the positively oriented $x$-axis and the vector $\overrightarrow{A_{i-1} A_{i}}$ is denoted by $\phi_{i}(i=1,2, \ldots n)$, then obviously $l_{1}\left(e_{i}\right)=l_{2}\left(e_{i}\right) \cdot\left(\left|\cos \phi_{i}\right|+\right.$ $\left.\left|\sin \phi_{i}\right|\right)$, while $l_{1}\left(e_{i}, \alpha\right)=l_{2}\left(e_{i}\right) \cdot\left(\left|\cos \left(\phi_{i}+\alpha\right)\right|+\left|\sin \left(\phi_{i}+\alpha\right)\right|\right)$. Thus, by using $1 \leq|\cos \beta|+|\sin \beta| \leq \sqrt{2}$ (for any $\beta$ ), we have

$$
\mathcal{P}_{2}(P) \leq \mathcal{P}_{1}(P, \alpha)=\sum_{i=1}^{n} l_{2}\left(e_{i}\right) \cdot\left(\left|\cos \left(\phi_{i}+\alpha\right)\right|+\left|\sin \left(\phi_{i}+\alpha\right)\right|\right) \leq \sqrt{2} \cdot \mathcal{P}_{2}(P) .
$$

We will exploit the following property of rectilinear polygons which is formulated as a theorem.

Theorem 1. A given polygon $P$ is rectilinear if and only if there exists a choice of the coordinate system such that the Euclidean perimeter of $P$ and the $l_{1}$ perimeter of $P$ coincide, i.e., 


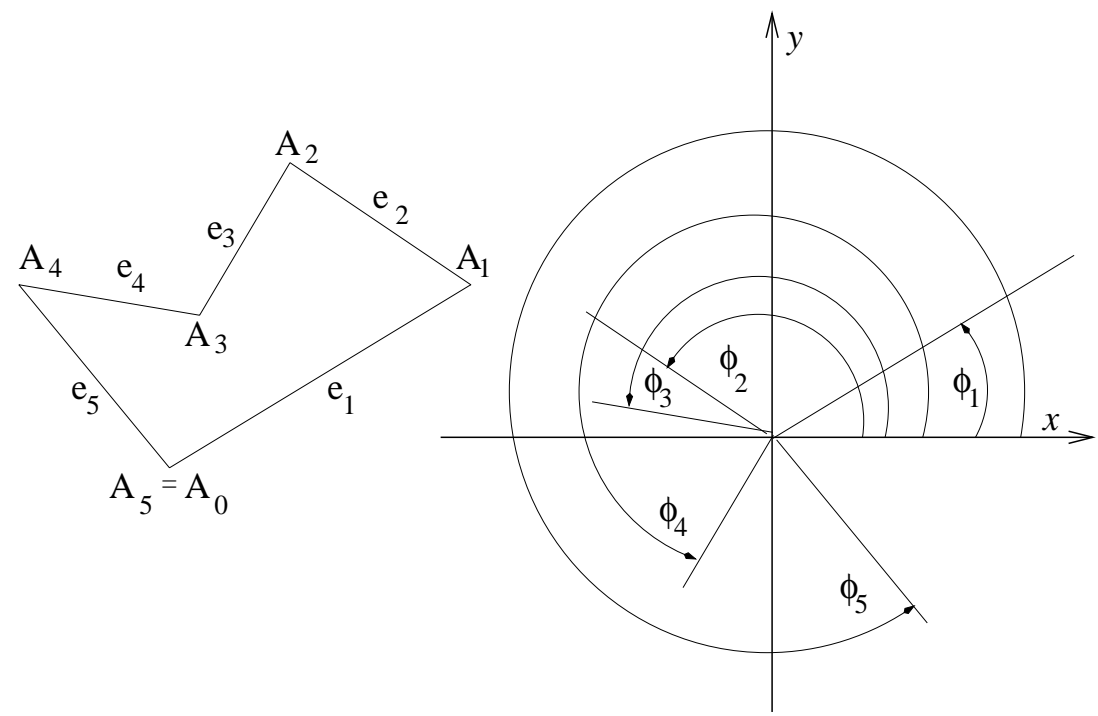

Fig. 3. The used denotations are illustrated on an example of a given 5-gon.

$$
\mathcal{P}_{2}(P)=\mathcal{P}_{1}(P, \alpha) \quad \text { for some } \alpha .
$$

Proof. If the given polygon $P$ is rectilinear, then a rotation of $P$, such that the edges of $P$ become parallel to the coordinate axes, preserves the equality $\mathcal{P}_{2}(P)=\mathcal{P}_{1}(P, \alpha)$ where $\alpha$ is the rotation angle.

On the other hand, if $\mathcal{P}_{2}(P)=\mathcal{P}_{1}(P, \alpha)$ then (by using (1) and $|\cos \beta|+$ $|\sin \beta| \geq 1)$ it must be $\left|\cos \left(\phi_{i}+\alpha\right)\right|+\left|\sin \left(\phi_{i}+\alpha\right)\right|=1$ for any edge $e_{i}, 1 \leq i \leq n$, of the given $n$-gon $P$. That means (for any $i, 1 \leq i \leq n$ ) it is either $\cos \left(\phi_{i}+\alpha\right)=0$ or $\sin \left(\phi_{i}+\alpha\right)=0$ - but it implies that all edges of $P$ are either parallel or orthogonal to the same line. This completes the proof.

\section{The Basic Idea and Necessary Mathematics}

Theorem 1 gives a useful characterisation of rectilinear polygons and gives the basic idea for the polygon rectilinearity measurement described in this paper. In the first stage, Theorem 1 together with $\mathcal{P}_{2}(P) \leq \mathcal{P}_{1}(P)$ (see (1)) suggests that the ratio $\frac{\mathcal{P}_{2}(P)}{\mathcal{P}_{1}(P)}$ can be used as a rectilinearity measure for the polygon $P$. Precisely, the ratio $\frac{\mathcal{P}_{2}(P)}{\mathcal{P}_{1}(P)}$ has the following "good" properties:

a1) it is a strictly positive number;

a2) it is defined for any polygon $P$;

a3) it can be calculated easily;

a4) for any non rectilinear polygon it is strictly less than 1 and for any rectilinear polygon it is exactly 1 if the coordinate axes are suitably chosen. 
But, on the other side $\frac{\mathcal{P}_{2}(P)}{\mathcal{P}_{1}(P)}$ has the following "bad" properties:

b1) it is not invariant under similarity (even isometric) transformations;

b2) the infimum for the set of values of $\mathcal{Q}(P, \alpha)=\frac{\mathcal{P}_{2}(P)}{\mathcal{P}_{1}(P, \alpha)}$ is not zero; for an example, it can be seen easily (from (1)) that there no exists a polygon $P$ such that $\frac{\mathcal{P}_{2}(P)}{\mathcal{P}_{1}(P)} \notin\left(0, \frac{\sqrt{2}}{2}\right)$.

In this section we develop necessary mathematical tools in order to define a function $\mathcal{R}(P)$ which satisfies a1)-a5) but not b1) and b2).

The problem described by b1) can be avoided by considering $\max _{\alpha \in[0,2 \pi]} \frac{\mathcal{P}_{2}(P)}{\mathcal{P}_{1}(P, \alpha)}$ instead of $\frac{\mathcal{P}_{2}(P)}{\mathcal{P}_{1}(P)}$, but it opens the question of how to compute this maximum.

Further, the inequalities from (1) give

$$
\frac{\sqrt{2}}{2} \leq \frac{\mathcal{P}_{2}(P)}{\mathcal{P}_{1}(P)} \leq 1, \quad \text { and consequently, } \quad \frac{\sqrt{2}}{2} \leq \max _{\alpha \in[0,2 \pi]} \frac{\mathcal{P}_{2}(P)}{\mathcal{P}_{1}(P, \alpha)} \leq 1
$$

for any polygon $P$. But, while the inequality $\frac{\mathcal{P}_{2}(P)}{\mathcal{P}_{1}(P)} \leq 1$ is sharp, and moreover, the equality $\max _{\alpha \in[0,2 \pi]} \frac{\mathcal{P}_{2}(P)}{\mathcal{P}_{1}(P, \alpha)}=1$ is satisfied if and only if $P$ is a rectilinear polygon (due to Theorem 1), it can be seen easily that there exists no polygon $P$ such that $\max _{\alpha \in[0,2 \pi]} \frac{\mathcal{P}_{2}(P)}{\mathcal{P}_{1}(P, \alpha)}=\frac{\sqrt{2}}{2}$. Namely, if an $n$-gon $\mathrm{P}$ satisfies the last equality, then for some $\alpha_{0}$ we have $\frac{\mathcal{P}_{2}(P)}{\mathcal{P}_{1}\left(P, \alpha_{0}\right)}=\frac{\sqrt{2}}{2}$ which (by (1)) would imply $\sqrt{2} \cdot l_{2}\left(e_{i}\right)=l_{1}\left(e_{i}, \alpha_{0}\right)$ or, equivalently, $\phi_{i}+\alpha_{0} \in\{\pi / 4,3 \pi / 4,7 \pi / 2,9 \pi / 4\}$ for any edge $e_{i}$ where $1 \leq i \leq n$. Thus, $P$ must be rectilinear, and due to Theorem 1 , the considered maximum must be equal to 1 , which is a contradiction.

So, for our purpose it is necessary to determine the maximal possible $\mu$ such that $\max _{\alpha \in[0,2 \pi]} \frac{\mathcal{P}_{2}(P)}{\mathcal{P}_{1}(P, \alpha)}$ belongs to the interval $[\mu, 1]$ for any polygon $P$. The next two lemmas together show $\mu=\frac{\pi}{4}$ and moreover, there is no polygon $P$ such that $\max _{\alpha \in[0,2 \pi]} \frac{\mathcal{P}_{2}(P)}{\mathcal{P}_{1}(P, \alpha)}=\frac{\pi}{4}$.

Lemma 1. The inequality

$$
\max _{\alpha \in[0,2 \pi]} \frac{\mathcal{P}_{2}(P)}{\mathcal{P}_{1}(P, \alpha)}>\frac{\pi}{4}
$$

holds for any polygon $P$. 
Proof. We prove the statement by a contradiction. Let us assume the contrary, i.e., there exists an $n$-gon $P$ such that

$$
\frac{\mathcal{P}_{2}(P)}{\mathcal{P}_{1}(P, \alpha)} \leq \frac{\pi}{4}
$$

for any choice of $\alpha$, or equivalently,

$$
\frac{\mathcal{P}_{1}(P, \alpha)}{\mathcal{P}_{2}(P)} \geq \frac{4}{\pi} \quad \text { for any } \quad \alpha \in[0,2 \pi] .
$$

Since $\frac{\mathcal{P}_{1}(P, \alpha)}{\mathcal{P}_{2}(P)}$ is a continuous non constant function (for more details see Section 5 ) depending on $\alpha$ (if $P$ is fixed), and since it is assumed to be greater or equal to $\frac{4}{\pi}$ it must be

$$
\int_{0}^{2 \pi} \frac{\mathcal{P}_{1}(P, \alpha)}{\mathcal{P}_{2}(P)} \cdot d \alpha>\int_{0}^{2 \pi} \frac{4}{\pi} \cdot d \alpha=8 .
$$

By using (2) we have:

$$
\begin{aligned}
8 & <\int_{0}^{2 \pi} \frac{\mathcal{P}_{1}(P, \alpha)}{\mathcal{P}_{2}(P)} \cdot d \alpha \\
& =\frac{1}{\mathcal{P}_{2}(P)} \cdot \int_{0}^{2 \pi}\left(\sum_{i=1}^{n} l_{1}\left(e_{i}, \alpha\right)\right) \cdot d \alpha \\
& =\frac{1}{\mathcal{P}_{2}(P)} \cdot \sum_{i=1}^{n}\left(\int_{0}^{2 \pi} l_{1}\left(e_{i}, \alpha\right) \cdot d \alpha\right) \\
& =\frac{1}{\mathcal{P}_{2}(P)} \cdot \sum_{i=1}^{n}\left(\int_{0}^{2 \pi} l_{2}\left(e_{i}\right) \cdot\left(\left|\sin \left(\phi_{i}+\alpha\right)\right|+\left|\cos \left(\phi_{i}+\alpha\right)\right|\right) \cdot d \alpha\right) \\
& =\frac{1}{\mathcal{P}_{2}(P)} \cdot\left(\sum_{i=1}^{n} 8 \cdot l_{2}\left(e_{i}\right)\right)=\frac{1}{\mathcal{P}_{2}(P)} \cdot \mathcal{P}_{2}(P) \cdot 8=8 .
\end{aligned}
$$

The contradiction $8<8$ finishes the proof. [

So, in accordance with the above discussion, Lemma 1 shows that the required number $\mu$ is not smaller than $\frac{\pi}{4}$. The next lemma shows that $\mu$ is not bigger than $\frac{\pi}{4}$. Thus, both lemmas together give $\mu=\frac{\pi}{4}$. 
Lemma 2. The infimum for the set of values $\max _{\alpha \in[0,2 \pi]} \frac{\mathcal{P}_{2}(P)}{\mathcal{P}_{1}(P, \alpha)}$ for all possible choices of polygon $P$ is $\frac{\pi}{4}$, i.e.,

$$
\inf _{P \in \Pi}\left\{\max _{\alpha \in[0,2 \pi]} \frac{\mathcal{P}_{2}(P)}{\mathcal{P}_{1}(P, \alpha)}\right\}=\frac{\pi}{4}
$$

Proof. To prove the statement it is enough to find a sequence of polygons $P_{3}, P_{4}, P_{5}, \ldots$ such that

$$
\lim _{n \rightarrow \infty}\left(\max _{\alpha \in[0,2 \pi]} \frac{\mathcal{P}_{2}\left(P_{n}\right)}{\mathcal{P}_{1}\left(P_{n}, \alpha\right)}\right)=\frac{\pi}{4} .
$$

We will prove that the sequence of regular $n$-gons $P_{n}$ inscribed into the unit circle satisfies the previous equality. Namely, it can be seen easily that the sequence of the Euclidean perimeters of $P_{n}$ tends to the perimeter of the unit circle, i.e.,

$$
\lim _{n \rightarrow \infty} \mathcal{P}_{2}\left(P_{n}\right)=2 \cdot \pi
$$

but also

$$
\lim _{n \rightarrow \infty} \mathcal{P}_{1}\left(P_{n}, \alpha\right)=8
$$

holds independently on the choice of $\alpha$. Precisely, if it is considered that (for any $\alpha \in[0,2 \pi])$ the $l_{1}$ perimeter $\mathcal{P}_{1}\left(P_{n}, \alpha\right)$ equals the sum of the projections of all edges of $P_{n}$ onto $x$ and $y$ axes, than it follows that this sum tends to 8 as $n \rightarrow \infty$ (see Fig. 4 for an illustration). Since the limits in (3) and (4) are independent on $\alpha$ we have

$$
\lim _{n \rightarrow \infty} \frac{\mathcal{P}_{2}\left(P_{n}\right)}{\mathcal{P}_{1}\left(P_{n}, \alpha\right)}=\lim _{n \rightarrow \infty}\left(\max _{\alpha \in[0,2 \pi]} \frac{\mathcal{P}_{2}\left(P_{n}\right)}{\mathcal{P}_{1}\left(P_{n}, \alpha\right)}\right)=\frac{\pi}{4}
$$

what finishes the proof. [

\section{A Rectilinearity Measure}

Motivated by the proved properties of the function $\max _{\alpha \in[0,2 \pi]} \frac{\mathcal{P}_{2}(P)}{\mathcal{P}_{1}(P, \alpha)}$ we give the following definition for the new rectilinearity measurement of polygons.

Definition 1. For an arbitrary polygon $P$ we define its rectilinearity $\mathcal{R}(P)$ as

$$
\mathcal{R}(P)=\frac{4}{4-\pi} \cdot\left(\max _{\alpha \in[0,2 \pi]} \frac{\mathcal{P}_{2}(P)}{\mathcal{P}_{1}(P, \alpha)}-\frac{\pi}{4}\right) .
$$

The following theorem summarises the properties of the polygon rectilinearity measure proposed here.

Theorem 2. For any polygon $P$, we have: 


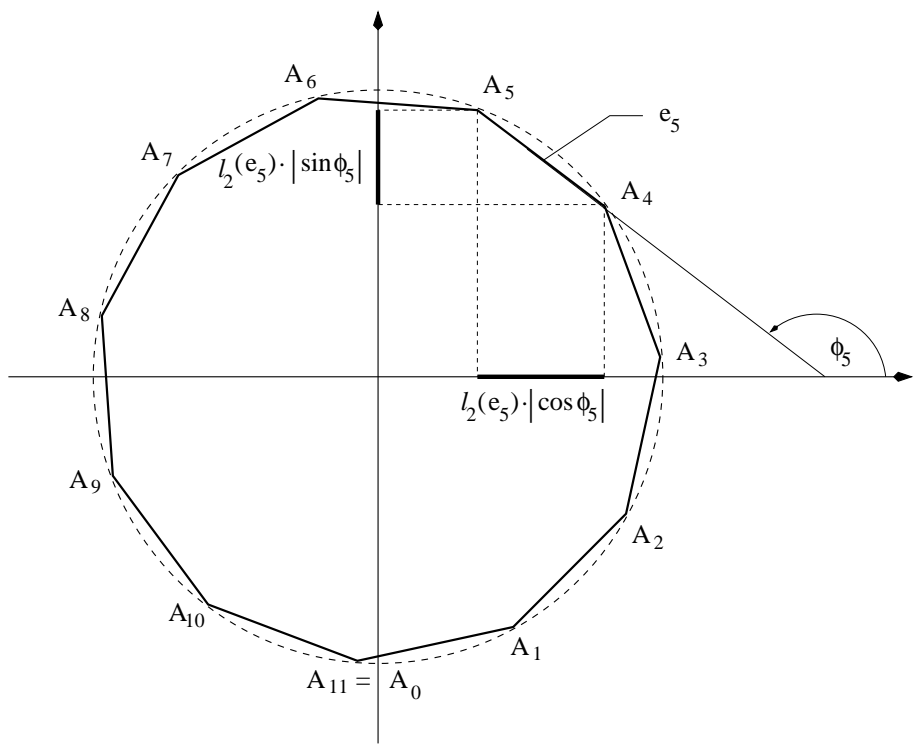

Fig. 4. A regular 11-gon inscribed into the unit circle with the centre placed at the origin.

i) $\mathcal{R}(P)$ is well defined and $\mathcal{R}(P) \in(0,1]$;

ii) $\mathcal{R}(P)=1$ if and only if $P$ is rectilinear;

iii) $\inf _{P \in \Pi}(\mathcal{R}(P))=0$;

iv) $\mathcal{R}(P)$ is invariant under similarity transformations.

Proof. For a fixed $P, \frac{\mathcal{P}_{2}(P)}{\mathcal{P}_{1}(P, \alpha)}$ is a continuous function depending on $\alpha$ (for more details see the next section). Consequently, it must reach its minimum on the closed interval $[0,2 \pi]$, i.e., $\mathcal{R}(P)$ is well defined. $\mathcal{R}(P) \in(0,1]$ follows from (1), Definition 1, and Lemma 1.

The item ii) is a direct consequence of Theorem 1.

The item iii) is the statement of Lemma 2.

To prove $i v$ ) let us notice that $\mathcal{R}(P)$ is invariant under all isometric transformations - which follows from the definition. Also, $\frac{\mathcal{P}_{2}(P)}{\mathcal{P}_{1}(P, \alpha)}$ and consequently $\mathcal{R}(P)$ are invariant under any transformation of the form $(x, y) \rightarrow(\lambda \cdot x, \lambda \cdot y)$ for any choice of $\lambda \neq 0, P$, and $\alpha$. That completes the proof. [

Some examples of polygons with their computed rectilinearity are given on Fig. 5 


\section{Computation of $\mathcal{R}(P)$}

The question which remains open is how to compute $\mathcal{R}(P)$ effectively if a polygon $P$ is given. Since $\mathcal{P}_{2}(P)$ can be easily calculated from the vertices of $P$ it remains to describe the computation of the minimum value of $\mathcal{P}_{1}(P, \alpha)$ when $\alpha$ varies from 0 to $2 \pi$. In this section we describe a procedure for such a computation.

Let us consider an edge $e_{i}(1 \leq i \leq n)$ of a given $n$-gon $P$. Trivially,

$$
l_{1}\left(e_{i}, \alpha\right)=\left\{\begin{array}{l}
+l_{2}\left(e_{i}\right) \cdot \cos \left(\phi_{i}+\alpha\right)+l_{2}\left(e_{i}\right) \cdot \sin \left(\phi_{i}+\alpha\right) \text { if } \alpha \in\left[-\phi_{i}, \frac{\pi}{2}-\phi_{i}\right] \\
-l_{2}\left(e_{i}\right) \cdot \cos \left(\phi_{i}+\alpha\right)+l_{2}\left(e_{i}\right) \cdot \sin \left(\phi_{i}+\alpha\right) \text { if } \alpha \in\left[\frac{\pi}{2}-\phi_{i}, \pi-\phi_{i}\right] \\
-l_{2}\left(e_{i}\right) \cdot \cos \left(\phi_{i}+\alpha\right)-l_{2}\left(e_{i}\right) \cdot \sin \left(\phi_{i}+\alpha\right) \text { if } \alpha \in\left[\pi-\phi_{i}, \frac{3 \pi}{2}-\phi_{i}\right] \\
+l_{2}\left(e_{i}\right) \cdot \cos \left(\phi_{i}+\alpha\right)-l_{2}\left(e_{i}\right) \cdot \sin \left(\phi_{i}+\alpha\right) \text { if } \alpha \in\left[\frac{3 \pi}{2}-\phi_{i}, 2 \pi-\phi_{i}\right] .
\end{array}\right.
$$

Consequently, there is an integer $k \leq 4 \cdot n$ and an increasing sequence of angles $0 \leq \alpha_{1}<\alpha_{2}<\ldots<\alpha_{k} \leq 2 \pi$ such that

$$
\mathcal{P}_{1}(P, \alpha)=\left\{\begin{array}{l}
\sum_{i=1}^{n} a_{1, i} \cdot \cos \left(\phi_{i}+\alpha\right)+b_{1, i} \cdot \sin \left(\phi_{i}+\alpha\right) \text { if } \alpha \in\left[\alpha_{1}, \alpha_{2}\right] \\
\sum_{i=1}^{n} a_{2, i} \cdot \cos \left(\phi_{i}+\alpha\right)+b_{2, i} \cdot \sin \left(\phi_{i}+\alpha\right) \text { if } \alpha \in\left[\alpha_{2}, \alpha_{3}\right] \\
\ldots \ldots \ldots \\
\sum_{i=1}^{n} a_{k-1, i} \cdot \cos \left(\phi_{i}+\alpha\right)+b_{k-1, i} \cdot \sin \left(\phi_{i}+\alpha\right) \text { if } \alpha \in\left[\alpha_{k-1}, \alpha_{k}\right] \\
\sum_{i=1}^{n} a_{k, i} \cdot \cos \left(\phi_{i}+\alpha\right)+b_{k, i} \cdot \sin \left(\phi_{i}+\alpha\right) \text { if } \alpha \in\left[\alpha_{k}, 2 \pi+\alpha_{1}\right],
\end{array}\right.
$$

where

$$
\left\{a_{i, j}, b_{i, j} \mid 1 \leq i \leq k, 1 \leq j \leq k\right\} \subset\left\{+l_{2}\left(e_{i}\right),-l_{2}\left(e_{i}\right)\right\},
$$

or precisely, for any $i \in\{1,2, \ldots, n\}$ and any $j \in\{1,2, \ldots, k\}$

$$
\begin{array}{llll}
a_{j, i}=+l_{2}\left(e_{i}\right) & \text { if } \quad \cos \left(\phi_{i}+\alpha\right)>0 & \text { for } \alpha \in\left(\alpha_{j}, \alpha_{j+1}\right) \\
a_{j, i}=-l_{2}\left(e_{i}\right) & \text { if } \quad \cos \left(\phi_{i}+\alpha\right)<0 & \text { for } \alpha \in\left(\alpha_{j}, \alpha_{j+1}\right)
\end{array}
$$

and analogously,

$$
\begin{array}{llll}
b_{j, i}=+l_{2}\left(e_{i}\right) & \text { if } \quad \sin \left(\phi_{i}+\alpha\right)>0 & \text { for } \alpha \in\left(\alpha_{j}, \alpha_{j+1}\right) \\
b_{j, i}=-l_{2}\left(e_{i}\right) & \text { if } \quad \sin \left(\phi_{i}+\alpha\right)<0 & \text { for } \alpha \in\left(\alpha_{j}, \alpha_{j+1}\right)
\end{array}
$$

What is important for us is

$$
0<\mathcal{P}_{1}(P, \alpha)=-\mathcal{P}_{1}^{\prime \prime}(P, \alpha) \quad \text { if } \quad \alpha \notin\left\{\alpha_{1}, \alpha_{2}, \ldots, \alpha_{k}\right\}
$$

which implies that $\mathcal{P}_{1}(P, \alpha)$ does not have any local minimum inside the open intervals

$$
\left.\left(\alpha_{1}, \alpha_{2}\right),\left(\alpha_{2}, \alpha_{3}\right), \ldots,\left(\alpha_{k-1}, \alpha_{k}\right),\left(\alpha_{k}, 2 \pi+\alpha_{1}\right)\right) .
$$

In other words we have

$$
\min _{\alpha \in[0,2 \pi]} \mathcal{P}_{1}(P, \alpha)=\min \left\{\mathcal{P}_{1}\left(P, \alpha_{1}\right), \mathcal{P}_{1}\left(P, \alpha_{2}\right), \ldots, \mathcal{P}_{1}\left(P, \alpha_{k}\right)\right\} .
$$


Now, we are able to describe the following simple procedure for computing $\mathcal{R}(P)$.

\section{PROCEDURE $\mathcal{R}(P)$ Computation}

Input: The vertices $A_{1}, A_{2}, \ldots, A_{n}$ of a given $n$-gon $P$.

\section{Step.}

For any $i, i=1,2, \ldots n$, compute the angle $\phi_{i}$, and compute the absolute values of the following angle differences:

$$
\left|\phi_{i}-\frac{\pi}{2}\right|,\left|\phi_{i}-\pi\right|,\left|\phi_{i}-\frac{3 \pi}{2}\right|,\left|\phi_{i}-2 \pi\right| .
$$

Sort the above angle-values in the increasing order and denote the sorted values as: $0 \leq \alpha_{1} \leq \alpha_{2}<\ldots<\alpha_{k} \leq 2 \pi$.

\section{Step.}

Assign either $+l_{2}\left(e_{i}\right)$ or $-l_{2}\left(e_{i}\right)$ to $a_{i, j}$ and $b_{i, j}$ for any $i$ from $\{1,2, \ldots, n\}$ and any $j$ from $\{1,2, \ldots, k\}$

( $\star$ in accordance with $(5)-(8) \star)$.

\section{Step.}

Compute $\min _{\alpha \in[0,2 \pi]} \mathcal{P}_{1}(P, \alpha)$ as the minimum of the finite number point set

$\left\{\mathcal{P}_{1}\left(P, \alpha_{1}\right), \mathcal{P}_{1}\left(P, \alpha_{2}\right), \ldots \mathcal{P}_{1}\left(P, \alpha_{k-1}\right), \mathcal{P}_{1}\left(P, \alpha_{k}\right)\right\}$

( $\star$ in accordance with $(9) \star$ )

then compute $\mathcal{R}(P)$ as

$$
\mathcal{R}(P):=\frac{4}{4-\pi} \cdot\left(\frac{\mathcal{P}_{2}(P)}{\min _{\alpha \in[0,2 \pi]} \mathcal{P}_{1}(P, \alpha)}-\frac{\pi}{4}\right)
$$

( $\star$ in accordance with Definition $1 \star$ ).

\section{Output: $\mathcal{R}(P)$.}

\section{Some Examples and Concluding Remarks}

The rectilinearity measure is applied to a (perfect) rectilinear polygon in the top left hand polygon in Fig. 5 which is then degraded in various ways. The first row demonstrates the effect of increasing levels of local noise applied to the polygon's vertices. In the second row the polygon is edited, eliminating vertices, which effectively rounds corners and increases its convexity. A shearing transformation is applied in the third row. Finally, the polygon is warped, and the axis aligned edges are increasingly rounded. All examples show that the rectilinearity measure is well behaved; increasing distortion consistently decreases the computed value. Note also that the orientations that maximised $\mathcal{Q}(P, \alpha)=\frac{\mathcal{P}_{2}(P)}{\mathcal{P}_{1}(P, \alpha)}$ match our 


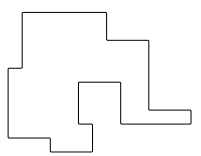

1.000

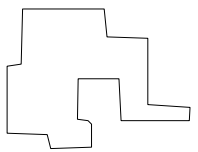

0.860

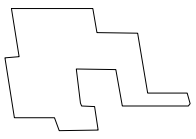

0.668

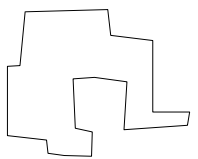

0.748

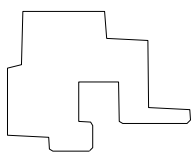

0.849

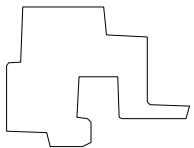

0.813

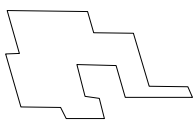

0.516

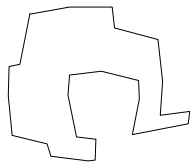

0.457

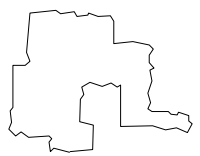

0.334

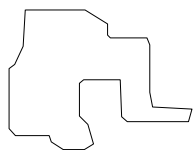

0.603

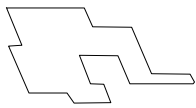

0.425

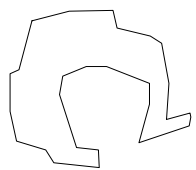

0.287

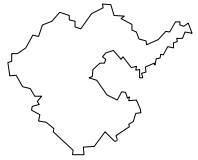

0.041

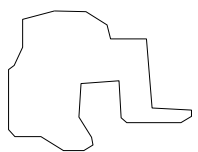

0.508

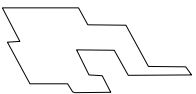

0.380

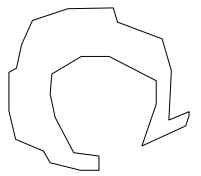

0.204

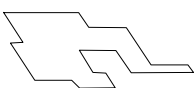

0.351

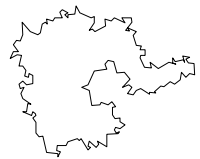

0.038

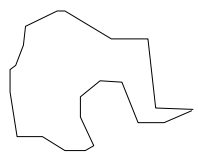

0.277

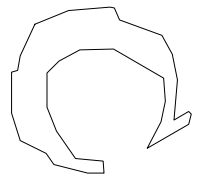

0.134

Fig. 5. Examples of polygons with their rectilinearity measured as proposed in this paper. Polygons are rotated to the orientations that maximised $\mathcal{Q}(P, \alpha)$.
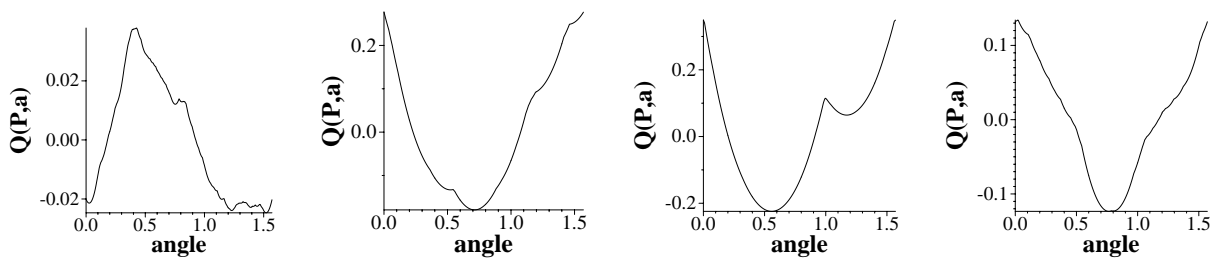

Fig. 6. Plots of normalised $\mathcal{Q}(P, \alpha)$ for each of the rightmost examples in Fig. 5 .

expectations except at high noise levels when the rectilinearity measure has dropped close to zero. For each of the maximally degraded polygons (i.e. the rightmost examples in each row) Fig. 6] plots $\mathcal{Q}(P, \alpha)$. It can be seen that it is well behaved and, despite the effects of noise and other distortions which introduce local maxima, the main peak remains distinct.

A second example is shown, working this time with real data from a Digital Elevation Model (DEM), i.e. a depth map (see Fig 7 (a)). Some simple noise filtering and segmentation techniques were applied to produce a set of polygons. These are further processed using Ramer's line simplification algorithm [10] to reduce the effects of quantisation since at a pixel level a dense 4-connected curve 


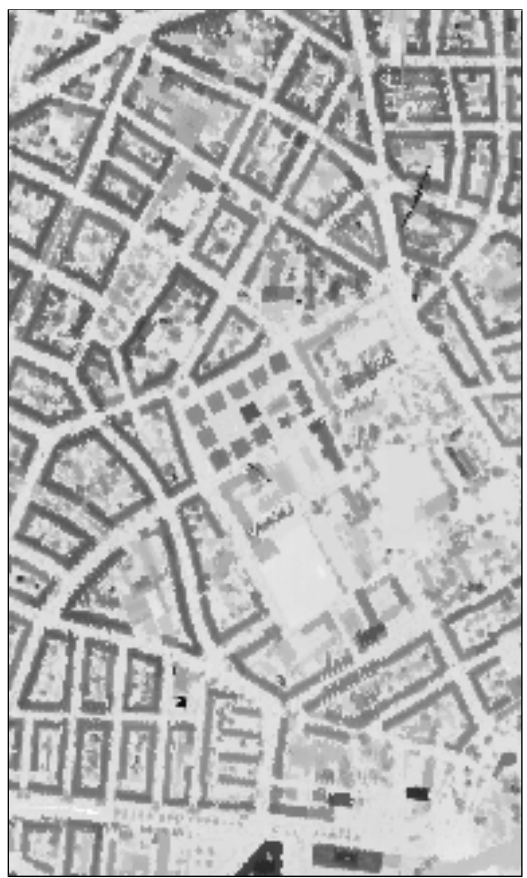

(a)

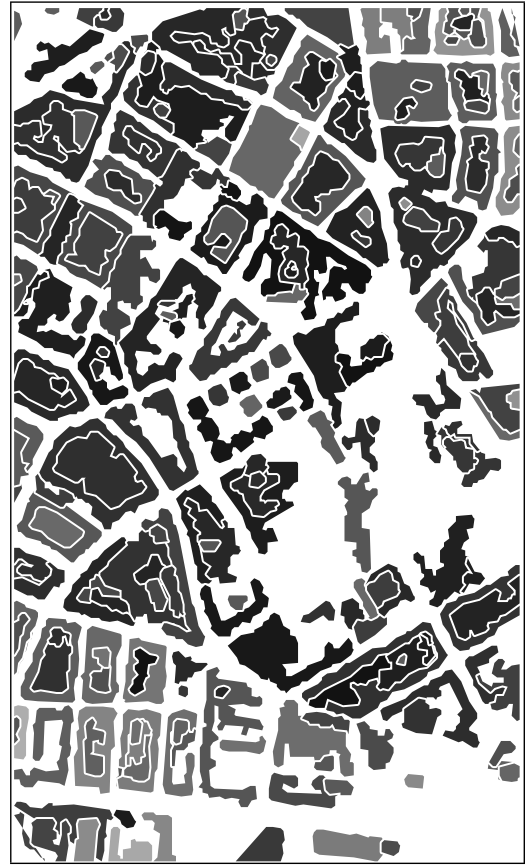

(b)

Fig. 7. (a) A DEM of Hannover; (b) segmented regions with filled intensities proportional to rectilinearity

will be perfectly rectilinear! Fig 7] (b) plots the regions filled with intensities proportional to their rectilinearity; thus rectilinear shapes should appear bright. A casual glance seems to indicate that in some cases the computed rectilinearity does not correspond well with human perception. As an example, the polygon making up the central building in Fig 7 (a) only receives a value of 0.087 although it initially looks approximately rectilinear. Nevertheless, when plotted again in Fig. 8 (a) at the orientation maximising $\mathcal{Q}(P, \alpha)$ the coordinate frame can be seen to be correctly determined. Moreover, the plot of $\mathcal{Q}(P, \alpha)$ (Fig. 8)(b) contains only the single main peak. In fact, the problem arises from errors in the boundary due to the various steps in data processing. The deviations of local edge orientation are substantial as demonstrated by a histogram of their orientation weighted by edge length. Fig. 8 (c) shows the histogram at two bin sizes, and it can be seen that the distribution is fairly uniform, therefore producing a low rectilinearity value.

As a possible means of reconciling this inconsistency between our perceptual and measured evaluation of rectilinearity we note that relatively small adjustments to the polygon could greatly increase the values of $\mathcal{Q}(\mathcal{P})$. Thus we intend to apply such a process, moving vertices such that they maximise $\mathcal{Q}(\mathcal{P})$ while minimising the shift in vertex location. A similar scheme of "shape correction" 


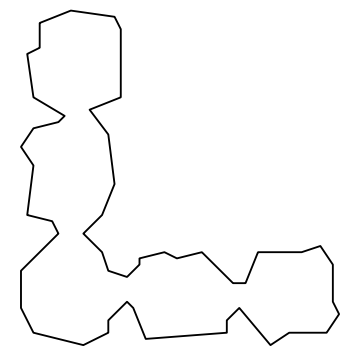

(a)

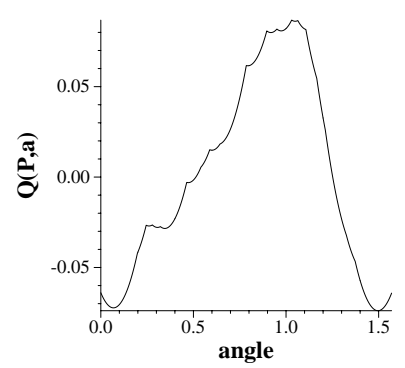

(b)

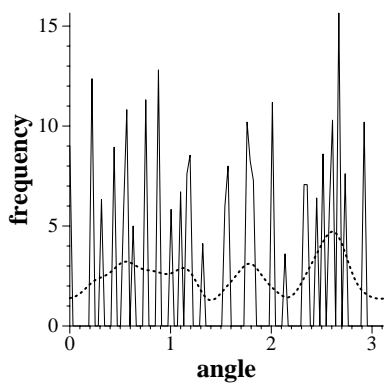

(c)

Fig. 8. (a) An example building rotated to maximise $\mathcal{Q}(P, \alpha)$; (b) the corresponding plot of $\mathcal{Q}(P, \alpha) ;(\mathrm{c})$ histogram of length weighted edge orientation of (a).

has already been applied by Brunn et al. [1] who applied an iterative MDL vertex shifting and removal scheme, and by Mayer [7] who used constrained snakes.

\section{References}

1. A. Brunn, U. Weidner, and W. Förstner. Model-based 2d-shape recovery. In G. Sagerer et al, editor, Mustererkennung, pages 260-268. Springer-Verlag, 1995.

2. R.T. Collins, C.O. Jaynes, Y.Q. Cheng, X.G. Wang, F.R. Stolle, E.M. Riseman, and A.R. Hanson. The ascender system: Automated site modeling from multiple aerial images. Computer Vision and Image Understanding, 72(2):143-162, 1998.

3. S. Hyde et al. The Language of Shape. Elsevier, 1997.

4. R.M. Haralick. A measure for circularity of digital figures. IEEE Transactions on Systems, Man and Cybernetics, 4:394-396, 1974.

5. R.B. Irvin and D.M. McKeown. Methods for exploiting the relationship between buildings and their shadows in aerial imagery. IEEE Transactions on Systems, Man and Cybernetics, 19(6):1564-1575, 1989.

6. Y.T. Liow and T. Pavlidis. Use of shadows for extracting buildings in aerial images. Computer Vision, Graphics and Image Processing, 49(2):242-277, 1990.

7. S. Mayer. Constrained optimization of building contours from high-resolution ortho-images. In Proc. Int. Conf. Image Processing, 2001.

8. S. Noronha and R. Nevatia. Detection and modeling of buildings from multiple aerial images. IEEE Transactions on Pattern Analysis and Machine Intelligence, 23(5):501-518, 2001.

9. D. Proffitt. The measurement of circularity and ellipticity on a digital grid. Pattern Recognition, 15(5):383-387, 1982.

10. U. Ramer. An iterative procedure for the polygonal approximation of plane curves. Computer Graphics and Image Processing, 1:244-256, 1972.

11. P.L. Rosin. Measuring rectangularity. Machine Vision and Applications, 11:191196, 1999.

12. M. Sonka, V. Hlavac, and R. Boyle. Image Processing, Analysis, and Machine Vision. Chapman and Hall, 1993.

13. J. Willats. Art and Representation: New Principles in the Analysis of Pictures. Princeton University Press, 1997. 